\title{
Erratum: Host non-inflammatory neutrophils mediate the engraftment of bioengineered
} vascular networks

Ruei-Zeng Lin, Chin Nien Lee, Rafael Moreno-Luna, Joseph Neumeyer, Breanna Piekarski, Pingzhu Zhou, Marsha A. Moses, Monisha Sachdev, William T. Pu, Sitaram Emani and Juan M. Melero-Martin

Nature Biomedical Engineering 1, 0081 (2017); published 13 June 2017; corrected 26 June 2017.

In the version of this Article originally published, the $x$ axis label 'Days' was missing from Fig. 1e, and in Fig. 2b, the top-right flow cytometry plot was incorrect. These errors have now been corrected. 